\title{
Utilidad de la terapia ocupacional y abordaje multidisciplinar de la fibromialgia
}

\section{Sr. Editor:}

La fibromialgia es un síndrome doloroso crónico, de causa desconocida, cuya clínica se caracteriza por dolor musculoesquelético agudo generalizado'. Afecta a entre el uno y el cuatro por ciento de la población ${ }^{2}$, mayoritariamente a mujeres (relación 20/1), y supone un importante problema social, tanto por su impacto económico como por la afectación de la calidad de vida de quienes la padecen $^{3-5}$. El tratamiento actual es sólo paliativo, postulándose un abordaje multidisciplinario, dentro del cual tiene especial cabida la terapia ocupacional (TO) ${ }^{6}$.

En la Universidad de Castilla-La Mancha hay abierta una línea de investigación interdepartamental sobre fibromialgia, dentro de la que estamos los autores de esta carta. Recientemente, se ha realizado un pequeño estudio, en el área de salud de Talavera de la Reina (Toledo) para valorar el grado de conocimiento que tienen tanto el personal sanitario como un grupo de enfermos de fibromialgia acerca de la TO y su aplicación en dicha enfermedad. En total se entrevistó a 47 profesionales de Atención Primaria, 51 de Atención Especializada y 30 personas diagnosticadas de fibromialgia.

Un 94,9\% de los sanitarios afirmó conocer la TO -aunque sólo el 9,1\% conocía alguna técnica específica-, mientras que estos porcentajes fueron del $86,7 \%$ y $60,0 \%$ respectivamente en los pacientes. A la pregunta sobre la posible eficacia de la TO en el tratamiento de la fibromialgia, respondieron afirmativamente el $40,8 \%$ de los profesionales y el $66,7 \%$ de los pacientes. Dos tercios de los pacientes habían recibido tratamiento con TO alguna vez; de ellos, el 36,6\% afirmó sentir clara mejoría una vez iniciado el tratamiento, frente al $6,7 \%$ que dijo sentirse igual. A la baja valoración de la utilidad por los profesionales se une la opinión de un $20 \%$ de ellos de que la fibromialgia es una "invención" voluntaria de los afectados. A pesar de ello, la mayoría de los profesionales $(78,9 \%)$ aseguran que el terapeuta ocupacional debería formar parte de los equipos multidisciplinares de todos los centros asistenciales.

Parece claro que los profesionales no conocen las posibilidades de la TO, ni en la fibromialgia ni en ninguna de las demás patologías, con lo que estamos perdiendo una importante herramienta en el abordaje de pacientes complejos, como los afectos de fibromialgia, en cuyo tratamiento ha demostrado su utilidad ${ }^{7-10}$, igual que sugieren los pacientes entrevistados.

La presencia de terapeutas ocupacionales en nuestro sistema sanitario es prácticamente testimonial en la actualidad (sólo un profesional en toda el área de salud de Talavera), pero podría suponer una reducción del número de consultas en Atención Primaria -nivel asistencial donde consultan una y otra vez estos pacientes- y del consecuente gasto farmacéutico que generan ${ }^{11}$.

La intervención que se lleva a cabo en Andalucía, única comunidad con terapeutas en Atención Primaria, consiste en consultas a domicilio, donde se trabajan y entrenan las actividades básicas e instrumentales de la vida diaria, se aplican principios ergonómicos para la salud, se realizan adaptaciones en el entorno, elaboración y entrenamiento de productos de apoyo u orientación al cuidador principal. También se llevan a cabo consultorías con otros profesionales del equipo o realización de actividades de promoción de salud y prevención de la enfermedad ${ }^{12}$. Los equipos multidisciplinares en el abordaje de la fibromialgia han logrado mejorías significativas en los dominios de buenos sentimientos, el control del dolor, la calidad de la fatiga, la rigidez en sus vidas, el catastrofismo y pruebas físicas ${ }^{13-15}$.

A la espera de los equipos multidisciplinares, el apoyo a los médicos de familia de terapeutas ocupacionales, fisioterapeutas o psicólogos, podría suponer un importante avance en la atención a estos pacientes.

Jaime González González', Olga Vicente Micó², Juan José Criado Álvarez ${ }^{3}$

${ }^{1}$ Especialista en Medicina Familiar y Comunitaria. Centro de Salud "La Solana" (Talavera de la

Reina). Profesor asociado de la Universidad de

Castilla la Mancha.

Email: jaimeg@sescam.jccm.es.

${ }^{2}$ Graduada en Terapia Ocupacional (CEU Talavera de la Reina).

${ }^{3}$ Especialista en Medicina Preventiva y Salud Pública. Profesor asociado de la Universidad de

CLM. 


\section{BIBLIOGRAFÍA}

1. Yunus MB, Masi AT, Aldag JC. A controlled study of fibromyalgia syndrome: clinical features and association with other functional syndromes. J Rheumatol Suppl. 1989;19:62-71.

2. McBeth J, Jones K. Epidemiology of chronic musculoskeletal pain. Best Pract Res Clin Rheumatol 2007;21:403-25.

3. Junyent PriuM, Benavides Ruiz M, Borrel Muñoz M, Siso Almiral A, Herrero Camp J. Fibromialgia: un reto para la Atención Primaria. Semergen. 2005;31:255-8.

4. Wolfe F, Anderson J, Harkness D, Bennett RM, Caro XJ, Goldenberg DL, Russell IJ, Yunus MB. A prospective, longitudinal, multicenter study of service utilization and costs in fibromyalgia. Arthritis Rheum. 1997;40(9):1560-70.

5. White K, Speecheley M, Harth M, Ostbye T. The London fibromylgia epidemiology study: Direct health care costs of fibromyalgia syndrome in London, Canada. J Rheumatol 1999; 26: 885-9.

6. Brown GT, Greenwood-Klein J. Juvenile fibromyalgia syndrome: the role for occupational therapists. Australian Occupational Therapy Journal.2001; 48(2): 54-65.

7. Villanueva VL, Valía JC, Cerdá G, Monsalve V, Bayona MJ, de Andrés J. Fibromialgia: diagnóstico y tratamiento. El estado de la cuestión. Rev Soc Esp Dolor. 2004;11:430443.

8. Romero Ayuso DM, Cenjor Rodríguez V, Cabello López A, Ureña A. Revista Asturiana de Terapia Ocupacional. 2006;3: 4-10.
9. Casso G, Cachin C, van Melle G, Gerster JC. Return- towork status 1 year after muscle reconditioning in chronic low back pain patients. Joint Bone Spine. 2004;71(2):1369.

10. Sim J, Adams N. Therapeutic approaches to fibromyalgia syndrome in the United Kingdom: a survey of occupational therapists and physical therapists. Eur J Pain. 2003;7:17380.

11. El dolor en la prensa. Rev Soc Esp Dolor [online] 2009;16:417-418.

12. Iglesias Arrabal C, Neira-Reina F, Pino Algarrada R, Elorza Guisasola J, Rendón Acosta A, Gómez Rodríguez F. Documento de consenso multidisciplinar para la implantación del Proceso Asistencial Integrado Fibromialgia en el área hospitalaria dependiente del Hospital Universitario de Puerto Real. Rev Soc Esp Dolor [online]. 2011; 18(4):241-248. Disponible en: http:// scielo.isciii.es/scielo.php?script=sci_arttext\&pid=S113480462011000400006\&lng=es.

13. Klyman CM, Browne M, Austad C, Spindler EJ, Spindler AC. A workshop model for educating medical practitioners about optimal treatment of difficult-tomanage patients: utilization of transference-countertransference. J Am Acad Psychoanal Dyn Psychiatry. 2008 ;36:661-76.

14. Huynh CN, Yanni LM, Morgan LA. Fibromyalgia: diagnosis and management for the primary healthcare provider. $\mathrm{J}$ Womens Health (Larchmt). $2008 ; 17: 1379-87$.

15. Schnur S. Primary fibromyalgia syndrome-diagnostic statistics from primary care. Z Rheumatol. 1992;51(3):11520. 